\title{
Anti-inflammatory and immunomodulatory effects of rosuvastatin in patients with low-to-moderate cardiovascular risk
}

\author{
ANA VAVLUKIS 1 ,* \\ MARIJA VAVLUKIS ${ }^{2}$ \\ ALEKSANDAR DIMOVSKI $^{1}$ \\ GORDANA PETRUSHEVSKA ${ }^{1}$ \\ ALEKSANDAR EFTIMOV ${ }^{2}$ \\ SASHKA DOMAZETOVSKA ${ }^{2}$ \\ KRISTINA MLADENOVSKA ${ }^{1}$ \\ ${ }^{1}$ University Ss Cyril and Methodius \\ Faculty of Pharmacy \\ 1000 Skopje, RN Macedonia \\ ${ }^{2}$ University Ss Cyril and Methodius \\ Faculty of Medicine \\ 1000 Skopje, RN Macedonia
}

Accepted June 21, 2021

Published online June 23, 2021

\begin{abstract}
Statins have shown anti-inflammatory pleiotropic effects in subjects with/at risk of cardiovascular disease. The aim of this study was to evaluate the inflammatory/immunomodulatory properties of rosuvastatin in subjects at low-tomoderate cardiovascular risk. Data was collected from patients' records, physical examination and blood sampling. Subjects were assigned to rosuvastatin $20 \mathrm{mg}$ per day. Rosuvastatin significantly decreased C-reactive protein $(p=0.045)$, and increased vascular endothelial growth factor $(p=0.004)$ and epidermal growth factor $(p=0.009)$. A multivariate analysis identified total cholesterol $(p=0.027)$ and vascular endothelial growth factor $(p=0.011)$ to be independently associated with rosuvastatin treatment. Given beneficial/ harmful role of growth factors, vascular endothelial growth factor (VEGF) and epidermal growth factor (EGF), in cardiovascular disease, one would suggest the need for routine monitoring of growth factor levels, especially in patients on long-term statin therapy.
\end{abstract}

Keywords: rosuvastatin, vascular endothelial growth factor, epidermal growth factor, cardiovascular risk

Cardiovascular disease (CVD) is still the major morbidity/mortality instigator in the world, despite improvements in the outcomes (1). The CVD precursor, atherosclerosis, is known to be present with chronic inflammation of the large and medium-sized arteries. Pathological studies have shown the predominance of immune/inflammatory cells, such as neutrophils, monocytes, and T- and B-cells, in, and adjunct to atherosclerotic lesions (2), as a result of endothelial dysfunction and increased endothelial adhesion (3). Different inflammatory cells produce a number of chemokines and cytokines, leading to smooth muscle cells (SMCs) dysfunction (3). After the inflammatory burst, numerous entities at the lesion site boost the on-going process, such as adipokines, immune complexes and reactive oxygen species (ROSs). Having this in mind, various inflammatory, immune, and oxidative biomarkers may help in identifying patients at CVD risk, and in monitoring treatment efficacy as well.

\footnotetext{
*Corresponding author: e-mail: ana.vavlukis@gmail.com
} 
Multiple risk factor intervention trial (MRFIT), the women's health study (WHS) and the emerging risk factor collaboration (ERFC) studies all showed a correlation between increased C-reactive protein (CRP) levels and increased CVD mortality, with a prediction power of CRP greater than that with low-density lipoprotein cholesterol (LDL-C) (4-6). While high-sensitivity (hs)CRP is a clinically proven risk assessment tool, still, this biomarker itself is not a sufficient marker of the cardiovascular (CV) inflammatory cascade as a whole. The sub-endothelial inflammation activates various immune cells to produce pro-inflammatory molecules, such as interleukin-1 (IL-1), IL-6, tumor necrosis factor- $\alpha$ (TNF- $\alpha)$, and monocyte chemo-attractant protein-1 (MCP-1), resulting in monocyte recruitment and macrophage activation (7). MCP-1 is one of the major regulators of vascular endothelial growth factor (VEGF) and epidermal growth factor (EGF), both cytoprotective and pro-angiogenic molecules (8). Therefore, all of the aforementioned inflammatory and immune mediators may have a potential role as a CVD biomarker and/or biomarker of treatment/ prevention efficacy.

Despite novel treatments, 3-hydroxy-3-methylglutaryl coenzyme A (HMG-CoA) reductase inhibitors, commonly known as statins, are still the golden clinical standard in primary and secondary CVD prevention (1). In addition to their anti-lipid effects, the anti-inflammatory/immunomodulatory properties of statins also account for their beneficial effects in subjects with/at risk of CVD. Statins increase nitric oxide levels in the endothelium, inhibit the nuclear factor kappa B (NF-kB) activation path, suppress ROS production and alteration of the vascular redox state, decrease endothelial adhesion and chemotaxis, and reduce MCP-1 expression (9). Evidence for these effects has been mainly gathered from CVD patients.

Although statin use is fundamental in CVD patients, questions still arise regarding their role in primary CVD prevention in people without an established disease. Despite the previous, current clinical practice guidelines encourage statin use in primary CV prevention (1). Besides LDL-C reduction, statins have many lipid-independent, pleiotropic effects, such as anti-inflammatory, antioxidant and immunomodulatory (10). Although studies have shown that statins act on both the innate and adaptive immune responses (11), human trials are lacking, especially in terms of CVD-free subjects.

Taken together, the aim of the presented study was to evaluate the anti-inflammatory and immunomodulatory properties of rosuvastatin in subjects at low-to-moderate cardiovascular risk, without an established CVD. The existing and emerging biomarkers of rosuvastatin response were evaluated and discussed, thus contributing to the global assessment of cardiovascular biomarkers suitability for diagnosis and treatment efficacy monitoring.

\section{EXPERIMENTAL}

\section{Subjects and study design}

For the purpose of the study, 55 adult outpatients (without an established CVD) [age $52.5 \pm 10.7$ year, $33(60 \%)$ women, $22(40 \%)$ men] from the UKIM-University Clinic of Cardiology in Skopje were included. Subjects were statin "naïve", with primary hypercholesterolemia or mixed dyslipidemia. Regarding CV risk-factors, the distribution of the subjects is presented in Table I.

The study was conducted in accordance with the ethical principles of the Declaration of Helsinki and the International Conference on Harmonization (ICH) Note for Guidance 
A. Vavlukis et al:: Anti-inflammatory and immunomodulatory effects of rosuvastatin in patients with low-to-moderate cardiovascular risk, Acta Pharm. 72 (2022) 303-315.

Table I. General characteristics of the study population

\begin{tabular}{ccc}
\hline \multirow{2}{*}{ Variable } & \multicolumn{2}{c}{ Percentage } \\
\cline { 2 - 3 } & Males & Females \\
\hline Hypertension & 73 & 67 \\
Diabetes mellitus type 2 & 18 & 27 \\
Pre-diabetes & 36 & 61 \\
Hyperlipidemia & 64 & 61 \\
Smoking & 14 & 21 \\
Family history of CVD & 9 & 18 \\
\hline
\end{tabular}

on Good Clinical Practice (GCP) (CPMP/ICH/135/95). All participants received oral and written information and gave a written consent before entering the study. The final clinical trial protocol, as well as the informed consent and other information that required preapproval, were reviewed, and approved by the local Ethics Committee for Human Research (University ss Cyril and Methodius, Faculty of Medicine (Skopje, RN Macedonia).

Data for the study inclusion was obtained from the patients' medical history, physical examination, and blood samples taken on the day of the clinical visit, with standard hematologic, electrolyte, protein indices and thyroid function measures used to determine the overall inclusion status of potential subjects. Subjects with known hypersensitivity to rosuvastatin or some of the formulation excipients, diagnosed with thyroid, liver, kidney and heart diseases, systemic inflammatory diseases, McArdle's disease, cancer history (remission shorter than 5 years), myopathy, rhabdomyolysis or muscle pain of unknown origin, human immune-deficiency virus (HIV), pregnant and nursing women or women planning to get pregnant, blood-donors (4 weeks before initiation of therapy), subjects with alanine transferase (ALT) and aspartate transferase (AST) levels 1.5-fold above the upper limit, increased creatine kinase (CPK) levels 5-fold above the upper limit, and subjects with creatinine clearance below $30 \mathrm{~mL} \mathrm{~min}^{-1}$ were not included in the study. Other exclusion criteria included prior treatment with lipid-lowering drugs, drugs known to affect cytochrome P450 3A4 and solute carrier organic anion transporter family member $1 B 1$ (OATP1B1) activity, and drugs known to increase LDL-C.

Subjects were assigned to rosuvastatin $20 \mathrm{mg}$ per day. It was considered that the patients adhered to the drug treatment when $80 \%$ of the dosage units were used. For determination of biochemical and hematological data, including inflammatory and immune markers, $10.0 \mathrm{~mL}$ venous blood in two EDTA/K3 vacuum tubes was taken from each patient at study entry, after 12 weeks of rosuvastatin treatment (efficacy indicators), and 4 weeks after drug discontinuation (safety indicators). Samples were taken from each subject at baseline and on-treatment, thus each subject acted as its own control. Samples were immediately transported (by a chain maintaining a $4{ }^{\circ} \mathrm{C}$ temperature of the samples) and analyzed after sampling. Biochemical parameters were determined at the UKIM-University Clinic of Clinical Biochemistry, Skopje, and the inflammatory markers were determined at the Institute of Pathology, UKIM-Faculty of Medicine, Skopje. In addition, total CVD risk, es expressed by SCORE values, SCORE risk age and SCORE relative risk, was also calculated at the baseline and after 12 weeks of treatment. 


\section{Biochemical and hematological profile}

Blood samples for the biochemical profile were collected in the morning, after an overnight fast, ca. 12 hours after the last dose. The response to rosuvastatin was evaluated according to the percentage change in total cholesterol (Chol), low density lipoprotein cholesterol (LDL-C), high density lipoprotein cholesterol (HDL-C), triglycerides (TG), apolipoprotein A1 (ApoA1), apolipoprotein B (ApoB), and lipoprotein (a) (Lp(a). As safety profile indicators, aspartate transaminase (AST), alanine transaminase (ALT), gamma-glutamyl transferase (GGT), creatine phosphokinase (CPK), myoglobin, glucose, and glycosylated hemoglobin A1c (HbA1c) were determined. Standard laboratory procedures and methods for evaluation of the biochemical parameters were used (COBAS INTEGRA tests), established by the International Federation of Clinical Chemistry (IFCC), Société Francoise de Biologie Clinique (SFBC), Committee on Enzymes of the Scandinavian Society for Clinical Chemistry and Clinical Physiology (SCE) and Deutsche Gesellschaft fur Klinische Chemie (DGKC). Standard procedures and methods were also used for the hematological, electrolyte, protein indices and thyroid function measures.

\section{Inflammatory and immune markers}

A panel of 13 inflammatory/immune variables was measured, including interleukins (IL-1 $\alpha$, IL-1 $\beta$, IL-2, IL-4, IL-6, IL-8 and IL-10), cytokines [tumor necrosis factor- $\alpha$ (TNF- $\alpha$ )], interferon- $\gamma$ (IFN- $\gamma$ ), chemokines (monocyte chemoattractant protein-1 (MCP-1), growth factors (epidermal growth factor (EGF) and vascular endothelial growth factor (VEGF), and acute phase proteins [C-reactive protein, (CRP)]. Quantitative determination of the inflammatory markers was done using a commercial Randox cytokine \& growth factors array kit (Randox Laboratories Ltd., UK) allowing a simultaneous in vitro quantitative detection, based on a sandwich chemiluminescent immune test.

\section{Statistical analysis}

Continuous variables are expressed as mean value $\pm S D$, and categorical variables are expressed as absolute numbers. $t$-Test was used for the statistical analysis of the variables before and after treatment. Correlations and receiver operating characteristic-ROC curves were computed, and a multivariate logistic regression analysis was made. Results were considered statistically significant when $p \leq 0.05$. Data was analyzed using the IBM SPSS 19.0 statistical software.

\section{RESULTS AND DISCUSSION}

\section{Lipid-lowering efficacy and safety of rosuvastatin}

Mean changes in the lipoprotein profile, as well as in the primary clinical efficacy indicators, SCORE, SCORE risk age and SCORE relative risk, during the study period, are shown in Table II. As expected, rosuvastatin significantly decreased total cholesterol, LDL-C, non-HDL-C, ApoB and TG. It did not have an effect on HDL-C and Lp(a). The reduction in the lipid levels led to a significant reduction of the overall calculated CVD risk of the 
A. Vavlukis et al:: Anti-inflammatory and immunomodulatory effects of rosuvastatin in patients with low-to-moderate cardiovascular risk, Acta Pharm. 72 (2022) 303-315.

Table II. Lipoprotein profile and SCORE risk before and after treatment

\begin{tabular}{cccc}
\hline Variable & $\begin{array}{c}\text { Pre-treatment } \\
(\text { mean } \pm \text { SD) }\end{array}$ & $\begin{array}{c}\text { Post-treatment } \\
(\text { mean } \pm \text { SD })\end{array}$ & $\begin{array}{c}t \text {-test } \\
(p \text {-value })\end{array}$ \\
\hline SCORE risk age & $57.9 \pm 6.9$ & $56.4 \pm 8.5$ & 0.007 \\
SCORE value & $3.2 \pm 3.4$ & $2.5 \pm 2.7$ & 0.000007 \\
SCORE relative risk & $2.5 \pm 1.2$ & $1.7 \pm 0.7$ & 0.00001 \\
Chol (mmol L $\left.{ }^{-1}\right)$ & $5.9 \pm 1.3$ & $4.2 \pm 0.9$ & 0.000002 \\
LDL-C $\left(\mathrm{mmol} \mathrm{L}^{-1}\right)$ & $3.6 \pm 1.3$ & $2.1 \pm 0.9$ & 0.000003 \\
HDL-C $\left(\mathrm{mmol} \mathrm{L}^{-1}\right)$ & $1.4 \pm 0.4$ & $1.4 \pm 0.3$ & NS \\
Non-HDL-C $\left(\mathrm{mmol} \mathrm{L}^{-1}\right)$ & $4.6 \pm 1.3$ & $2.9 \pm 1.0$ & 0.00001 \\
TG (mmol L-1) & $2.0 \pm 1.1$ & $1.7 \pm 0.9$ & 0.003 \\
ApoA1 $\left(\mathrm{mg} \mathrm{L}^{-1}\right)$ & $1.6 \pm 0.3$ & $1.6 \pm 0.3$ & $\mathrm{NS}$ \\
ApoB $\left(\mathrm{mg} \mathrm{L}^{-1}\right)$ & $1.4 \pm 0.4$ & $0.9 \pm 0.3$ & 0.000478 \\
Lp(a) $(\mathrm{mg} \mathrm{per} \mathrm{100} \mathrm{mL)}$ & $53.5 \pm 85.5$ & $57.9 \pm 88.8$ & $\mathrm{NS}$ \\
\hline
\end{tabular}

$n=55$

SCORE - Systematic COronary Risk Evaluation (categories: low < 1; moderate 1-5; high 5-9; very high $\geq 10$ )

NS - not significant

study population, expressed by the SCORE values, SCORE risk age and SCORE relative risk (Fig. 1).

According to the vast clinical data on statins side-effects, safety of rosuvastatin treatment was determined by monitoring glycaemia and HbA1c, mean change in liver enzymes, and laboratory indicators of possible muscle toxicity. In Table III, the levels of the selected safety indicators in the study population, before and after rosuvastatin treatment,

Table III. Safety indicators before and after treatment

\begin{tabular}{cccc}
\hline Variable & $\begin{array}{c}\text { Pre-treatment } \\
(\text { mean } \pm \mathrm{SD})\end{array}$ & $\begin{array}{c}\text { Post-treatment } \\
(\text { mean } \pm \mathrm{SD})\end{array}$ & $\begin{array}{c}t \text {-test } \\
(p \text {-value })\end{array}$ \\
\hline AST $\left(\mathrm{U} \mathrm{L}^{-1}\right)$ & $23.1 \pm 7.1$ & $23.4 \pm 6.8$ & NS \\
ALT $\left(\mathrm{U} \mathrm{L}^{-1}\right)$ & $25.3 \pm 9.9$ & $26.1 \pm 10.8$ & NS \\
GGT $\left(\mathrm{U} \mathrm{L}^{-1}\right)$ & $21.2 \pm 7.3$ & $21.4 \pm 6.9$ & NS \\
CPK $\left(\mathrm{U} \mathrm{L}^{-1}\right)$ & $114.4 \pm 63.3$ & $123.7 \pm 87.3$ & NS \\
Myoglobin $\left(\mathrm{ng} \mathrm{mL}^{-1}\right)$ & $46.0 \pm 16.5$ & $46.6 \pm 16.4$ & NS \\
Glycaemia $\left(\mathrm{mmol} \mathrm{L}^{-1}\right)$ & $6.2 \pm 2.9$ & $5.7 \pm 2.0$ & $\mathrm{NS}$ \\
\hline
\end{tabular}

$n=55$

NS - not significant 


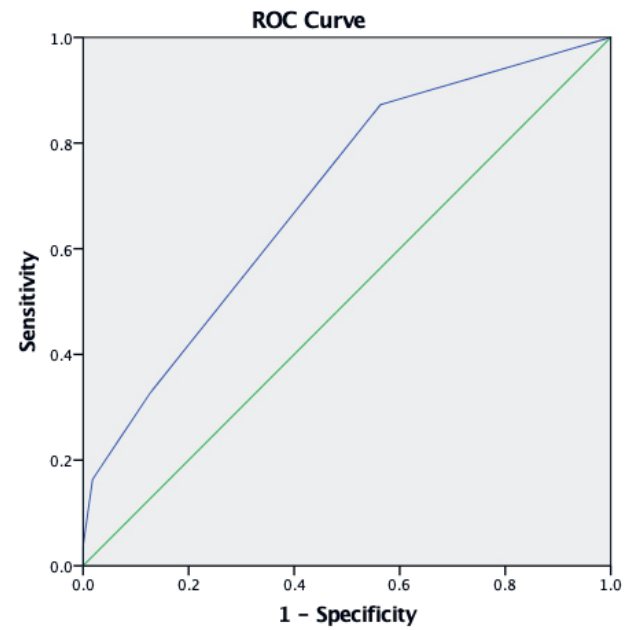

Diagonal segments are produced by ties.

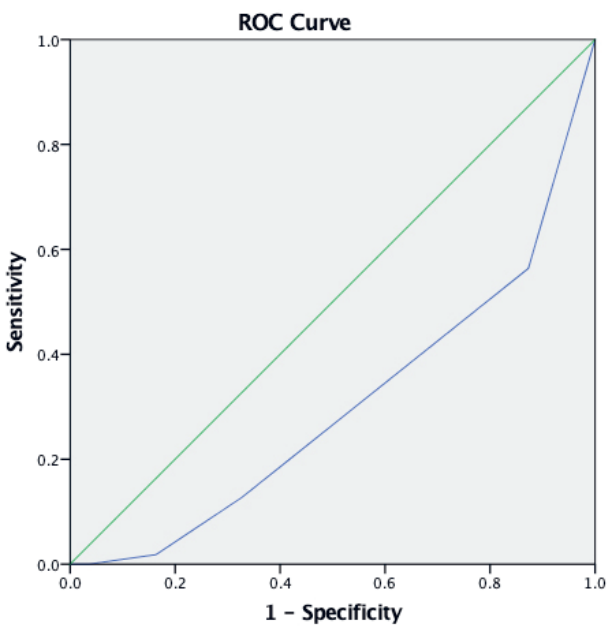

Diagonal segments are produced by ties.

\begin{tabular}{lccccc}
\hline & \multirow{2}{*}{ Area } & Standard error & Sig. ( $p$-value) & \multicolumn{2}{c}{ Asymptotic 95 \% CI } \\
\cline { 1 - 3 } Relative CVD risk & & & & Lower bound & Upper bound \\
\hline Before treatment (left) & 0.699 & 0.050 & 0.00007 & 0.602 & 0.796 \\
After treatment (right) & 0.301 & 0.050 & 0.00007 & 0.204 & 0.398 \\
\hline
\end{tabular}

CI - confidence interval

Fig. 1. Graphical plot (receiver operating characteristics - ROC curve) that demonstrates the therapeutic performance of rosuvastatin treatment on the relative CVD risk of the study population.

are presented. No significant changes in the safety indicators were observed after rosuvastatin treatment.

Other biochemical and hematological parameters were also determined, primarily to satisfy the requirements for inclusion in the study group. However, when the same parameters were evaluated after a 12-week rosuvastatin treatment, significant decrease in uric acid $(p=0.053)$, and thyroid-stimulating hormone (TSH) $(p=0.007)$ and increase in free thyroxine (FT4) $(p=0.013)$ levels was observed. Studies have found that statins reduce thyroid volume, and the prevalence, number and volume of nodules, even though this was only shown with long-term statin treatment -5 years or longer (12). Additionally, it was suggested that this apoptotic effect appears in patients with enlarged thyroid glands, not so much in those with a normal thyroid size, as a result of the mevalonate path prenylation inhibition. In that light it is thought that statins act on the pituitary gland to suppress TSH production, leading to decreased TSH levels, and thus decreased stimulation of the thyroid gland. One possible explanation of the observed association of rosuvastatin with the thyroid measures is that the statin-treatment resulting in decreased levels of TSH in fact stimulates the normally functioning thyroid gland to produce more T4, leading to increased 
concentrations, by the principle of the negative feedback regulation. Another thought is that rosuvastatin acts on the thyroid gland by stimulating T4 production as a primary mechanism, which by a negative feedback leads to decreased TSH levels.

Serum uric acid levels have been addressed as a marker of CV risk, since preclinical and clinical data has shown that patients with increased uric acid levels carry bigger risk of cardiac and renal damage, as well as CV events (13). Animal studies have proposed a renal protective effect of rosuvastatin, by reducing serum uric acid levels (14). The mechanism of this action is largely unknown.

\section{Inflammatory and immunomodulatory pleiotropic effects of rosuvastatin}

The levels of twelve inflammatory/immune markers, in addition to CRP-levels, were determined in the study population before and after rosuvastatin treatment. The measured values are presented in Table IV. As the data shows, rosuvastatin significantly decreased CRP, and increased VEGF and EGF. Rosuvastatin also led to an increase of several pro-inflammatory cytokines (TNF- $\alpha$, IL- $1 \alpha$ and IL-1 $\beta$ ), chemokines (IL-8, MCP-1) and type 2 inflammatory mediators (IL-4), as well as an increase of the central regulator of immune responses (IL-2). Also, a decrease of the pro-inflammatory cytokine IL-6 was observed. However, all these changes did not achieve statistical significance. In addition, no effect on the major effector of immunity, IFN- $\gamma$ level, was observed.

Studies on the effect of statins on different inflammatory/immunomodulating cytokines and chemokines, although modest in quantity, are conflicting. The majority of studies

Table IV. Inflammatory/immune marker levels before and after treatment

\begin{tabular}{cccc}
\hline Variable & $\begin{array}{c}\text { Pre-treatment } \\
(\text { mean } \pm \text { SD })\end{array}$ & $\begin{array}{c}\text { Post-treatment } \\
(\text { mean } \pm \text { SD })\end{array}$ & $\begin{array}{c}t \text {-test } \\
(p \text {-value })\end{array}$ \\
\hline CRP $\left(\mathrm{mg} \mathrm{L}^{-1}\right)$ & $3.0 \pm 3.6$ & $1.9 \pm 1.6$ & 0.045 \\
IL-2 $\left(\mathrm{pg} \mathrm{mL}^{-1}\right)$ & $1.9 \pm 2.4$ & $2.7 \pm 4.4$ & $\mathrm{NS}$ \\
IL-4 $\left(\mathrm{pg} \mathrm{mL}^{-1}\right)$ & $2.2 \pm 1.6$ & $2.5 \pm 1.0$ & $\mathrm{NS}$ \\
IL-6 $\left(\mathrm{pg} \mathrm{mL}^{-1}\right)$ & $4.6 \pm 23.1$ & $2.2 \pm 2.9$ & $\mathrm{NS}$ \\
IL-8 $\left(\mathrm{pg} \mathrm{mL}^{-1}\right)$ & $7.0 \pm 9.9$ & $12.4 \pm 22.1$ & $\mathrm{NS}$ \\
IL-10 $\left(\mathrm{pg} \mathrm{mL}^{-1}\right)$ & $0.8 \pm 1.0$ & $1.2 \pm 1.5$ & $\mathrm{NS}$ \\
IL-1 $\alpha\left(\mathrm{pg} \mathrm{mL}^{-1}\right)$ & $0.3 \pm 0.2$ & $0.5 \pm 0.8$ & $\mathrm{NS}$ \\
IL-1 $\left(\mathrm{pg} \mathrm{mL}^{-1}\right)$ & $2.0 \pm 3.3$ & $2.8 \pm 6.5$ & $\mathrm{NS}$ \\
IFN- $\gamma\left(\mathrm{pg} \mathrm{mL}^{-1}\right)$ & $1.0 \pm 4.8$ & $1.0 \pm 2.8$ & $\mathrm{NS}$ \\
TNF- $\alpha\left(\mathrm{pg} \mathrm{mL}^{-1}\right)$ & $2.4 \pm 1.8$ & $3.1 \pm 3.4$ & $\mathrm{NS}$ \\
MCP-1 $\left(\mathrm{pg} \mathrm{mL}^{-1}\right)$ & $106.0 \pm 79.3$ & $118.6 \pm 83.7$ & $\mathrm{NS}$ \\
VEGF $\left(\mathrm{pg} \mathrm{mL}^{-1}\right)$ & $42.8 \pm 41.8$ & $77.9 \pm 77.6$ & 0.004 \\
EGF $\left(\mathrm{pg} \mathrm{mL}^{-1}\right)$ & $65.1 \pm 77.8$ & $112.6 \pm 105.9$ & 0.009 \\
\hline
\end{tabular}

$n=55$

NS - not significant 
of ex vivo and in vivo treated leukocytes, have reported that statins reduce the levels of pro-inflammatory cytokines (15). On the contrary, ex vivo monocyte treatment with lipophilic statins (simvastatin, atorvastatin and lovastatin), but not with hydrophilic statins (pravastatin and rosuvastatin) stimulated the secretion of numerous cytokines, which could be blocked by mevalonic acid (16). Regarding rosuvastatin, studies have reported results to the opposite extremes - some that it can reduce TNF- $\alpha$, IL- 6 and IL-1 $\alpha$ (9), some that it does not have an effect on circulating levels of different inflammatory biomarkers (17); the others have even reported that rosuvastatin stimulates the production of both caspase-1-dependent (inflammasome) (IL-1 $\beta$, IL-18) and -independent (IFN- $\gamma$, TNF- $\alpha$, IL-5, IL-6, IL-15) cytokines (18). The latter is in line with our results. The reason for these divergent findings is mainly unclear, although it has been suggested that it may be dependent on the basal inflammatory condition of the subjects and/or cells studied (18).

Every measured variable was analyzed with a univariate binary logistic regression analysis, in order to detect possible statistically significant differences in variable levels associated with rosuvastatin (Table V). Rosuvastatin treatment was negatively correlated with SCORE relative risk, total cholesterol, LDL-C, non-HDL-C, ApoB and CRP, and positively correlated with FT4, VEGF and EGF. The discrimination abilities of the univariate analysis-identified variables were tested using receiver operating characteristics (ROC) curves (Fig. 2).

VEGF is a major angiogenic molecule controlling vascular homeostasis, growth and function, vascular permeability and vasodilatation, which makes it important in the process of neovascularization and angiogenesis (19). EGF is another cytoprotective, pro-angiogenic growth factor, necessary for cellular proliferation, differentiation and survival (19). Current reports have confirmed the presence of neocapillaries in plaques, suggesting that

Table V. Efect of rosuvastatin on the clinical risk profile, lipid, biochemical and inflammatory biomarkers calculated with a univariate binary logistic regression analysis ${ }^{a, b}$

\begin{tabular}{cccc}
\hline Variable & Beta & $p$-value & OR (95\% CI) \\
\hline Relative risk & -0.952 & 0.001 & $0.38(0.23-0.66)$ \\
Cholesterol & -1.320 & 0.00005 & $0.267(0.163-0.437)$ \\
LDL-C & -1.300 & 0.00007 & $0.273(0.163-0.455)$ \\
Non-HDL-C & -1.283 & 0.0007 & $0.277(0.170-0.451)$ \\
ApoB & -4.121 & 0.000008 & $0.016(0.003-0.084)$ \\
FT4 & 0.172 & 0.065 & $1.188(0.990-1.427)$ \\
CRP & -0.203 & 0.069 & $0.817(0.656-1.016)$ \\
VEGF & 0.010 & 0.008 & $1.010(1.003-1.018)$ \\
EGF & 0.006 & 0.014 & $1.006(1.001-1.011)$ \\
\hline
\end{tabular}

\footnotetext{
${ }^{a}$ Fifty-five patients.

${ }^{\mathrm{b}}$ This the test that determines the relationship between one independent variable (treatment with rosuvastatin), and one dependent variable. Listed are only the relationships with statistical significance.

OR - odds ratio, CI - confidence interval
} 


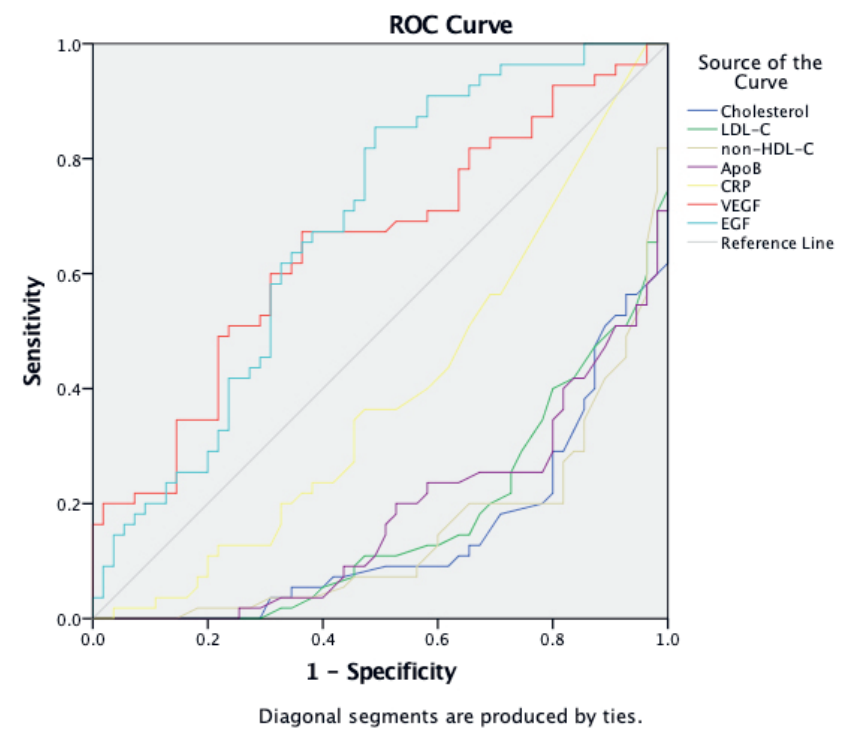

\begin{tabular}{|c|c|c|c|c|c|}
\hline \multirow{2}{*}{$\begin{array}{l}\text { Lipid and } \\
\text { inflammatory } \\
\text { biomarkers }\end{array}$} & \multirow{2}{*}{ Area } & \multirow{2}{*}{$\begin{array}{l}\text { Standard } \\
\text { error }\end{array}$} & \multirow{2}{*}{$\begin{array}{l}\text { Significance } \\
(p \text {-value })\end{array}$} & \multicolumn{2}{|c|}{ Asymptotic $95 \%$ CI } \\
\hline & & & & Lower Bound & Upper Bound \\
\hline Cholesterol & 0.147 & 0.035 & 0.00006 & 0.078 & 0.216 \\
\hline LDL-C & 0.171 & 0.038 & 0.00008 & 0.097 & 0.245 \\
\hline Non-HDL-C & 0.150 & 0.036 & 0.0006 & 0.079 & 0.221 \\
\hline ApoB & 0.181 & 0.039 & 0.0004 & 0.105 & 0.257 \\
\hline CRP & 0.396 & 0.054 & 0.059 & 0.289 & 0.502 \\
\hline VEGF & 0.648 & 0.053 & 0.007 & 0.545 & 0.751 \\
\hline EGF & 0.683 & 0.051 & 0.001 & 0.583 & 0.784 \\
\hline
\end{tabular}

CI - confidence interval

Fig. 2. Graphical plot (receiver operating characteristics - ROC curve) that demonstrates the therapeutic performance of rosuvastatin treatment on the lipid and inflammatory biomarkers of the study population.

neoangiogenesis plays a role in the progression of atherosclerotic plaques and subsequent complications (20). On the other hand, VEGF and EGF levels have been shown to be lower in stable coronary artery disease and ischemic heart disease patients, compared to healthy controls (19), which should propose benefit of higher growth factors levels for the reparation of diseased vessels.

Statins have shown biphasic effects on angiogenesis, as a result of contrary effects of low (nanomolar) vs. high (low micromolar, i.e., $1-10 \mu \mathrm{mol} \mathrm{L}{ }^{-1}$ ) drug concentrations (21). 
A. Vavlukis et al:: Anti-inflammatory and immunomodulatory effects of rosuvastatin in patients with low-to-moderate cardiovascular risk, Acta Pharm. 72 (2022) 303-315.

Table VI. Variables that were identified as independently associated with rosuvastatin treatment $t^{a, b}$

\begin{tabular}{cccccccc}
\hline & & & & & & \multicolumn{2}{c}{$95 \%$ CI for Exp(B) } \\
\cline { 6 - 8 } & & W & Wald & $p$-value & Exp(B) & Lower & Upper \\
\hline Step 1 & Relative risk & -0.428 & 1.886 & 0.170 & 0.652 & 0.354 & 1.201 \\
& Cholesterol & -0.447 & 0.323 & 0.570 & 0.639 & 0.137 & 2.992 \\
& LDL-C & -0.010 & 0.000 & 0.988 & 0.990 & 0.287 & 3.413 \\
& Non-HDL-C & -0.131 & 0.027 & 0.870 & 0.877 & 0.184 & 4.195 \\
& ApoB & -2.768 & 2.571 & 0.109 & 0.063 & 0.002 & 1.851 \\
& FT4 & 0.174 & 1.667 & 0.197 & 1.190 & 0.914 & 1.549 \\
& CRP & -0.121 & 1.008 & 0.315 & 0.886 & 0.699 & 1.122 \\
& VEGF & 0.013 & 4.035 & 0.045 & 1.013 & 1.000 & 1.026 \\
EGF & 0.003 & 0.591 & 0.442 & 1.003 & 0.996 & 1.009 \\
& Constant & 3.707 & 2.439 & 0.118 & 40.741 & & \\
& Cholesterol & -0.854 & 4.872 & 0.027 & 0.426 & 0.200 & 0.909 \\
& ApoB & -2.321 & 2.641 & 0.104 & 0.098 & 0.006 & 1.613 \\
& VEGF & 0.014 & 6.392 & 0.011 & 1.014 & 1.003 & 1.025 \\
& Constant & 6.096 & 20.802 & 0.000 & 443.933 & & \\
\hline
\end{tabular}

${ }^{a}$ Fifty-five patients.

${ }^{\mathrm{b}}$ Multivariate linear regression analysis, with a backward conditional method - in the first step all nine univariate variables were included in the model, in the final step only three variables remained in the model.

$\operatorname{Exp}(\mathrm{B})$ - exponentiation of the B coefficient, which is an odds ratio, CI - confidence interval

Low-dose statins are supposed to enhance angiogenesis via activation of the endothelial NO synthase. By contrast, high-dose statins are reported to decrease angiogenesis, due to a statin-induced reduction in the geranylgeranylation of the Rho and Ras proteins, which regulate the activity of the VEGF receptor (22).

Medium-dose simvastatin was reported to decrease VEGF levels in hypercholesterolemic subjects (23), atorvastatin treatment was shown to reduce EGF and VEGF levels in coronary artery disease (CAD) patients (24), and lovastatin has been reported to decrease both basal and cytokine-induced VEGF production (21). On the other hand, high-dose simvastatin and cerivastatin were reported to stimulate VEGF and EGF expression (23). Studies have reported opposite results regarding rosuvastatin effect on growth factor levels. Rosuvastatin showed a dose-dependent effect on EGF levels, with inhibition of EGF expression only with lower rosuvastatin concentrations (25). Yokohama et al. (26) reported decreased hepatic expression of VEGF and EGF in rosuvastatin-fed mice. Opposite to this, Cantoni et al. (27) reported that rosuvastatin promoted myocardial neovascularization in chronic heart failure patients, as a result of enhanced VEGF expression. A study on patients with mixed dyslipidemias showed a dose-dependent effect of rosuvastatin - highintensity rosuvastatin treatment (40 mg per day) reduced VEGF levels, whereas moderate-intensity rosuvastatin treatment (10 mg per day) led to a significant VEGF increase (28). 
Even though that we also used a high-intensity rosuvastatin treatment ( $20 \mathrm{mg}$ per day), we registered significant increases in both VEGF and EGF levels, which is in accordance with the results reported by Cantoni et al. (27), but is opposed to the results reported by Chantzichristos et al. (28).

The obtained results were confirmed with a multivariate analysis (multivariate logistic regression; model - backward conditional; characteristics of the model - chi-square 57.136, $p<0.0001)$, where two independent variables were identified: total cholesterol $(p=0.027)$ and VEGF ( $p=0.011)$, although ApoB remained in the model. As expected, total cholesterol was negatively associated, while VEGF remained positively associated with rosuvastatin treatment (Table VI).

In the light of such diverse findings, one crucial question to be answered is whether the clinical context (disease) of the patient, or the statin treatment intensity, is the main cursor that defines the direction of the changes in these growth factors.

\section{Limitations of the study}

The small sample size might be a reason for omitting possible statistically significant associations that would appear with a larger sample size.

\section{CONCLUSIONS}

We have executed an investigation of the inflammatory/ immunomodulatory, as well as lipid-modifying effects of rosuvastatin in subjects at low-to-moderate cardiovascular risk, without an established CVD. Of the thirteen examined biomarkers, despite CRP decrease, VEGF and EGF showed a strong statistically significant increase in response to rosuvastatin treatment in the studied population.

Determining whether the clinical settings of the patient, or the statin therapy duration and/or intensity defines the levels of VEGF and EGF seems pivotal in future investigations.

Acronyms, abbreviations, symbols. - ALT - alanine transferase, ApoA1 - apolipoprotein A1, ApoB - apolipoprotein B, AST - aspartate transferase, CAD - coronary artery disease, Chol - total Cholesterol, CPK - creatine kinase, CRP - C-reactive protein, CV - cardiovascular, CVD - Cardiovascular disease, DM - diabetes mellitus, EGF - epidermal growth factor, ERFC - Emerging risk factor collaboration trial, GGT - gamma-glutamyl transferase, HbA1c - glycosylated hemoglobin A1c, HDLC - high density lipoprotein cholesterol, HIV - human immune-deficiency virus, HLP - hyperlipidemia, HMG-CoA - 3-hydroxy-3-methylglutaryl coenzyme A, HTA - hypertension, IFN- $\gamma$ interferon- $\gamma$, IL - interleukin, LDL-C - low-density lipoprotein cholesterol, Lp(a) - lipoprotein (a), MCP-1 - monocyte chemoattractant protein-1, MRFIT - multiple risk factor intervention trial, NF-kB - nuclear factor kappa B, OATP1B1 - solute carrier organic anion transporter family member 1B1, ROSs - reactive oxygen species, SCORE - Systemic COronary Risk Evaluation, SMCs - smooth muscle cells, TG - triglyceride, TNF- $\alpha$ - tumor necrosis factor- $\alpha$, TSH - thyroid-stimulating hormone, VEGF - vascular endothelial growth factor, WHS - women's health study

Acknowledgements. - This research is part of a national clinical study entitled: "Rosuvastatin effects on lipoprotein proteomics in hyperlipidemic patients: new biomarker identification for treatment efficacy monitoring". The authors would also like to acknowledge BIOTEK Solutions for providing the Randox kit for the measurement of the inflammatory/immune markers. 


\section{REFERENCES}

1. M. F. Piepoli, A. W. Hoes, S. Agewall, C. Albus, C. Brotons, A. L. Catapano, M. Cooney, U. Corrà, B. Cosyns, C. Deaton, I. Graham, M. S. Hall, F. D. R. Hobbs, M. Løchen, H. Löllgen, P. Marques-Vidal, J. Perk, E. Prescott, J. Redon, D. J. Richter, N. Sattar, Y. Smulders, M. Tiberi, H. B. van der Worp, I. van Dis, W. M. M. Verschuren and S. Binno, 2016 European Guidelines on cardiovascular disease prevention in clinical practice: The sixth joint task force of the European Society of Cardiology and other societies on cardiovascular disease prevention in clinical practice (constituted by representatives of 10 societies and by invited experts) developed with the special contribution of the European Association for Cardiovascular Prevention \& Rehabilitation (EACPR), Eur. Heart J. 37 (2016) 23152381; https://doi.org/10.1093/eurheartj/ehw106

2. J. L. Fleg, G. W. Stone, Z. A. Fayad, J. F. Granada, T. S. Hatsukami, F. D. Kolodgie, J. Ohayon, R. Pettigrew, M. S. Sabatine, G. J. Tearney, S. Waxman, M. J. Domanski, P. R. Srinivas and J. Narula, Detection of high-risk atherosclerotic plaque: report of the NHLBI working group on current status and future directions, JACC Cardiovasc. Imag. 5 (2012) 941-955; https://doi.org/10.1016/j.jcmg.2012.07.007

3. P. Libby, P. M. Ridker and G. K. Hansson, Inflammation in atherosclerosis: From pathophysiology to practice, J. Am. Coll. Cardiol. 54 (2009) 2129-2138; https://doi.org/ 10.1016/j.jacc.2009.09.009

4. L. H. Kuller, R. P. Tracy, J. Shaten and E. N. Meilahn, Relation of C-reactive protein and coronary heart disease in the MRFIT nested case-control study. Multiple risk factor intervention trial, Am. J. Epidemiol. 144 (1996) 537-547; https://doi.org/ 10.1093/oxfordjournals.aje.a008963

5. P. M. Ridker, N. Rifai, L. Rose, J. E. Buring and N. R. Cook, Comparison of C-reactive protein and low-density lipoprotein cholesterol levels in the prediction of first cardiovascular events, N. Engl. J. Med. 347 (2002) 1557-1565; https://doi.org/ 10.1056/NEJMoa021993

6. S. Kaptoge, E. Di Angelantonio, G. Lowe, M. B. Pepys, S. G. Thompson, R. Collins and J. Danesht, C-reactive protein concentration and risk of coronary heart disease, stroke, and mortality: an individual participant meta-analysis, Lancet 375 (2010) 132-140; https://doi.org/ 10.1016/S01406736(09)61717-7

7. V. Lubrano and S. Balzan, Consolidated and emerging inflammatory markers in coronary artery disease, World J. Exp. Med. 5 (2015) 21-32; https://doi.org/ 10.5493/wjem.v5.i1.21

8. I. Subirana, M. Fitó, O. Diaz, J. Vila, A. Francés, E. Delpon, J. Sanchis, R. Elosua, D. Muñoz-Aguayo, I. R. Dégano and J. Marrugat, Prediction of coronary disease incidence by biomarkers of inflammation, oxidation, and metabolism, Sci. Rep. 8 (2018) Article ID 3191; https://doi.org/ 10.1038/s41598-01821482-y

9. E. Diamantis, G. Kyriakos, L. V. Quiles-Sanchez, P. Farmaki and T. Troupis, The anti-inflammatory effects of statins on coronary artery disease: An updated review of the literature, Curr. Cardiol. Rev. 13 (2017) 209-216; https://doi.org/ 10.2174/1573403X13666170426104611

10. F. Mach, C. Baigent, A. L. Catapano, K. C. Koskinas, M. Casula, L. Badimon, M. J. Chapman, G. G. De Backer, V. Delgado, B. A. Ference, I. M. Graham, A. Halliday, U. Landmesser, B. Mihaylova, T. R. Pedersen, G. Riccardi, D. J. Richter, M. S. Sabatine, M. Taskinen, L. Tokgozoglu and O. Wiklund, 2019 ESC/EAS guidelines for the management of dyslipidaemias: lipid modification to reduce cardiovascular risk: The task force for the management of dyslipidaemias of the European Society of Cardiology (ESC) and European Atherosclerosis Society (EAS), Eur. Heart J. 41 (2020) 111-188; https://doi. org/10.1093/eurheartj/ehz455

11. D. Tousoulis, C. Psarros, M. Demosthenous, R. Patel, C. Antoniades and C. Stefanadis, Innate and adaptive inflammation as a therapeutic target in vascular disease: the emerging role of statins, J. Am. Coll. Cardiol. 63 (2014) 2491-2502; https://doi.org/ 10.1016/j.jacc.2014.01.054

12. C. Demir, C. Anil, Y. Bozkus, U. Mousa, A. Kut, A. Nar and N. B. Tutuncu, Do statins affect thyroid volume and nodule size in patients with hyperlipidemia in a region with mild-to-moderate iodine deficiency? A Prospective Study, Med. Princ. Pract. 27 (2018) 1-7; https://doi.org/ 10.1159/000486748 
A. Vavlukis et al:: Anti-inflammatory and immunomodulatory effects of rosuvastatin in patients with low-to-moderate cardiovascular risk, Acta Pharm. 72 (2022) 303-315.

13. M. L. Muiesan, C. Agabiti-Rosei, A. Paini and M. Salvetti, Uric acid and cardiovascular disease: An update, Eur. Cardiol. 11 (2016) 54-59; https://doi.org/ 10.15420/ecr.2016:4:2

14. G. Derosa, P. Maffioli, Ž. Reiner, L. E. Simental-Mendía and A. Sahebkar, Impact of statin therapy on plasma uric acid concentrations: A systematic review and meta-analysis, Drugs 76 (2016) 947-956; https://doi.org/ 10.1007/s40265-016-0591-2

15. D. Ferro, S. Parrotto, S. Basili, C. Alessandri and F. Violi, Simvastatin inhibits the monocyte expression of proinflammatory cytokines in patients with hypercholesterolemia, J. Am. Coll. Cardiol. 36 (2000) 427-431; https://doi.org/ 10.1016/S0735-1097(00)00771-3

16. E. Mandosi, M. Fallarino, A. Gatti, A. Carnovale, M. Rossetti, E. Lococo, B. Buchetti, S. Filetti, L. Lenti and S. Morano, Atorvastatin downregulates monocyte CD36 expression, nuclear NFkB and TNF-a levels in type 2 diabetes, J. Atheroscler. Thromb. 17 (2010) 539-545; https://doi.org/ 10.5551/ jat.2956

17. T. Lyngdoh, P. Vollenweider, G. Waeber and P. M. Vidal, Association of statins with inflammatory cytokines: a population based Colaus study, Atherosclerosis 219 (2011) 253-258; https://doi.org/ 10.1016/j.atherosclerosis.2011.07.117

18. P. Karmaus, M. Shi, S. Perl, A. Biancotto, J. Candia, F. Cheung, Y. Kotliarov, N. Young and M. B. Fessler, Effects of rosuvastatin on the immune system in healthy volunteers with normal serum cholesterol, JCI Insight 4 (2019) e131530; https://doi.org/ 10.1172/jci.insight.131530

19. I. Karu, J. Starkopf, K. Zilmer and M. Zilmer, Growth factors serum levels in coronary artery disease patients scheduled for bypass surgery: Perioperative dynamics and comparisons with healthy volunteers, BioMed Res. Int. 2013 (2013) Article ID 985404; https://doi.org/10.1155/2013/985404

20. C. Camaré, M. Pucelle, A. Nègre-Salvayre and R. Salvayre, Angiogenesis in the atherosclerotic plaque, Redox Biol. 12 (2017) 18-34; https://doi.org/ 10.1016/j.redox.2017.01.007

21. J. Dulak and A. Józkowicz, Anti-angiogenic and anti-inflammatory effects of statins: relevance to anti-cancer therapy, Curr. Cancer Drug Targets 5 (2005) 579-594; https://doi.org/10.2174/156800905774932824

22. M. Weis, C. Heeschen, A. J. Glassford and J. P. Cooke, Statins have biphasic effects on angiogenesis, Circulation 105 (2002) 739-745; https://doi.org/ 10.1161/hc0602.103393

23. A. Giurgea, C. Margeta, T. Maca, A. Rezaie-Majd, R. A. Bucek, M. Manavi, R. Afarideh, E. Minar and M. Baghestanian, Simvastatin reduces serum level of vascular endothelial growth factor in hypercholesterolemic patients, J. Cardiovasc. Pharmacol. 47 (2006) 30-36; https://doi.org/ 10.1097/01. fjc.0000191970.65998.7a

24. H. F. Alber, J. Dulak, M. Frick, W. Dicht, S. P. Schwarzacher, O. Pachinger and F. Weidinger, Atorvastatin decreases vascular endothelial growth factor in patients with coronary artery disease, J. Am. Coll. Cardiol. 39 (2002) 1951-1955; https://doi.org/ 10.1016/S0735-1097(02)01884-3

25. V. Capra and G. E. Rovati, Rosuvastatin inhibits human airway smooth muscle cells mitogenic response to eicosanoid contractile agents, Pulm. Pharmacol. Ther. 27 (2014) 10-16; https://doi.org/ 10.1016/j.pupt.2013.06.005

26. K. Yokohama, S. Fukunishi, M. Ii, K. Nakamura, H. Ohama, Y. Tsuchimoto A. Asai, Y. Tsuda and K. Higuchi, Rosuvastatin as a potential preventive drug for the development of hepatocellular carcinoma associated with non-alcoholic fatty liver disease in mice, Int. J. Mol. Med. 38 (2016) 1499-1506; https://doi.org/ 10.3892/ijmm.2016.2766

27. S. Cantoni, C. Cavallini, F. Bianchi, F. Bonavita, V. Vaccari, E. Olivi, I. Frascari, R. Tassinari, S. Valente, V. Lionetti and C. Ventura, Rosuvastatin elicits KDR-dependent vasculogenic response of human placental stem cells through PI3K/AKT pathway, Pharmacol. Res. 65 (2012) 275-284; https://doi.org/ 10.1016/j.phrs.2011.12.004

28. V. G. Chantzichristos, A. P. Agouridis, E. Moutzouri, K. Stellos, M. S. Elisaf and A. D. Tselepis, Plasma VEGF and IL-8 levels in patients with mixed dyslipidaemia. Effect of rosuvastatin monotherapy or its combination at a lower dose with omega-3 fatty acids: A pilot study, Curr. Vasc. Pharmacol. 14 (2016) 474-480; https://doi.org/ 10.2174/1570161114666160404125341 